\title{
Design and control of system for elbow rehabilitation: Preliminary findings
}

\author{
Tadeusz Mikołajczyk ${ }^{1, A-F}$, Adam Kłodowski, ${ }^{2, A-F}$, Emilia Mikołajewska, ${ }^{3, B-F}$, Paweł Walkowiak ${ }^{1, B-F}$, Pedro Berjano ${ }^{4, A-F}$, \\ Jorge Hugo Villafañe ${ }^{5, A-F}$, Francesco Aggogeri ${ }^{6, B-F}$, Alberto Borboni ${ }^{6, B-F}$, Davide Fausti ${ }^{7, B-F}$, Gianluigi Petrogalli, ${ }^{7, B-F}$ \\ ${ }^{1}$ Department of Production Technology, Faculty of Mechanical Engineering, University of Science and Technology, Bydgoszcz, Poland \\ ${ }^{2}$ Laboratory of Machine Design, School of Energy Systems, Lappeenranta University of Technology, Finland \\ ${ }^{3}$ Department of Physiotherapy, Faculty of Health Sciences, Ludwik Rydygier Collegium Medicum in Bydgoszcz, Nicolaus Copernicus University in Toruń, Poland \\ ${ }^{4}$ GSpine4 Spine Surgery Division, Institute for Recovery and Care of Scientific Characteristics - Galeazzi Orthopedic Institute, Milan, Italy \\ ${ }^{5}$ Institute for Recovery and Care of Scientific Characteristics - Don Gnocchi Foundation, Milan, Italy \\ ${ }^{6}$ Applied Mechanics Research Group, Faculty of Engineering, University of Brescia, Italy \\ ${ }^{7}$ Research and Development Department, Polibrixia Innovation Engineering, Brescia, Italy \\ A - research concept and design; $\mathrm{B}$ - collection and/or assembly of data; $\mathrm{C}$ - data analysis and interpretation; \\ $D$ - writing the article; $E$ - critical revision of the article; $F$ - final approval of the article
}

Address for correspondence

Emilia Mikołajewska

E-mail: e.mikolajewska@wp.pl

Funding sources

None declared

Conflict of interest

None declared

Received on 0ctober 31, 2016

Reviewed on March 13, 2017

Accepted on June 8, 2017

DOI

\section{Abstract}

Background. The use of an exoskeleton elbow is considered an effective treatment in several pathologies, including post-stroke complications, traumatic brain injury (TBI) and spinal cord injury (SCI), as well as in patients with neurodegenerative disorders. The effectiveness of rehabilitation is closely linked to a suitably chosen therapy. The treatment can be performed only by specialized personnel, significantly supported with the use of automated devices.

Objectives. The aim of this study was to present a novel exoskeleton for elbow rehabilitation without a complicated control system.

Material and methods. Single-degree-of-freedom (SDOF) solution in constructing the prototype of an elbow exoskeleton for rehabilitation purposes has been applied. The simplicity of the actuation mechanism was set as one of the priorities in the design; thus, a single-axis stepper motor with a controller was found to be adequate for providing a reliable and precise source of motion for the exoskeleton.

Results. Technological development may provide novel solutions, such as an exoskeleton - a wearable, external structure which supports or (in selected applications) even replaces the muscle actuation in the patient. The reported advantages of the proposed exoskeleton reflect current state-of-the-art. The proposed control strategy relies on closed-loop position control, performance, low manufacturing cost, and predicted performance in a rehabilitation scenario. All these factors play an important role in establishing the directions for further research, e.g., an integrated force sensor in the device, measurements of torque interactions on the elbow joint, and assessment and response to an overload of articulation.

Conclusions. This study suggests not only the clinical but also the possible economic and logistical advantages offered by the portability of the system, and its effective support for therapists applying an elbow exoskeleton.

Key words: rehabilitation, assistive technology, elbow, exoskeleton, upper limb exoskeleton

10.17219/acem/74556

Copyright

Copyright by Author(s)

This is an article distributed under the terms of the

Creative Commons Attribution Non-Commercial License

(http://creativecommons.org/licenses/by-nc-nd/4.0/) 


\section{Introduction}

In Western countries, elderly people will suffer from a growing number of neuromotor disorders each year, and this age group is at greatest risk for disability. In that population, it is important that physically weak people are able to take care of themselves. ${ }^{1}$ The question of the superiority of robot training of the upper limbs over classical therapies in neuromotor disorder patients remains controversial. ${ }^{2-4}$ During the subacute stage, upper limbs training is likely to be the most useful.

Advances in technology have led to the development of a variety of robotic devices for the use in rehabilitation. Robotic devices have been progressively included in neurorehabilitation programs. ${ }^{5-8}$ Exoskeletons are wearable robots which exhibit a close cognitive and physical interaction with the human user. They are robotic exoskeletal structures that typically operate alongside human limbs. ${ }^{9}$ Scientific and technological work on exoskeletons began in the early 1960s, but only recently they have been applied to rehabilitation and functional substitution in patients suffering from motor disorders. ${ }^{10,11}$ The effectiveness of rehabilitation is closely linked with the suitability of the chosen therapy. ${ }^{12,13}$ Robotic techniques allow the precise recording of movements and the application of forces to the affected limb using visual cues; they convert repetitive movement practice into a useful task within everyday activity. ${ }^{14} \mathrm{Cur}$ rent state-of-the-art of robotic systems and their prospective function in the post-stroke rehabilitation of the upper limbs is presented in the studies conducted by Fausti et al., ${ }^{12}$ Bishop and Stein, ${ }^{15}$ Hochstenbach-Waelen et al., ${ }^{16}$ Loureiro et al., ${ }^{17}$ Lu et al., ${ }^{18}$ Maciejasz et al., ${ }^{19}$ and Morales et al. ${ }^{20}$ The application of robotics in neurorehabilitation is promising, but is still not widely used in clinical settings.

The aim of this study was to present a novel exoskeleton for elbow rehabilitation without a complicated control system.

\section{Challenges and requirements}

We took into consideration the needs and preferences of patients, their families and caregivers, and therapists when designing a robot supporting upper limb rehabilitation. A study by Lu et al. showed the main requirements for an upper limb rehabilitation device, including the following:

- facilitating a variety of arm movements;

- being usable in a seated position;

- giving feedback to clients;

- including virtual activities specific to daily living;

- being useful at home;

- having adjustable resistance, and

- costing less than 6000 USD. ${ }^{18,21}$

To sum up, based on the classification proposed by Maciejasz et al., ${ }^{19}$ we proposed a novel exoskeleton for upper limb rehabilitation purposes in low- and middle-income countries, with the following features:
- application field: supporting basic activities of daily living (ADL), neurological rehabilitation and orthopedic rehabilitation;

- target group: patients with severe neurological deficits, including post-stroke patients, those with traumatic brain injury (TBI) or spinal cord injury (SCI), geriatric patients with neurodegenerative disorders, and people who need similar solutions for functional support during recovery only (e.g., for avoiding physical effort after cardiopulmonary diseases);

- type of assistance: active device and elbow strength assistance;

- mechanical design: exoskeleton-based arm with a single one-degree-of-freedom (SDOF) stepper motor and control system;

- control strategy: a mix of kinematic and dynamic; and

- clinical evaluation: experimental study, further research and a randomized controlled trial.

The most common symptoms in elbow functional deficits are weakness, loss of joint control, excessive muscle contraction, spastic co-contraction, and pathological synergies. ${ }^{22-27}$ The most recent, state-of-the-art advances in the area of the elbow exoskeletons has been presented by Vitiello et al. ${ }^{28}$

An elbow exoskeleton combines motor (re)learning principles. It should provide repetition of task-related movements, tailored to the patient and the patient's goals, in a meaningful context, with associated variability and increasing levels of difficulty in exercises. The hardware and software should allow for easy use (including preparation for the exercises), and easy adjustment to individual patients' health status, needs and change over time (recovery or relapse). The important features are safety, price, proven efficacy, and motivation to exercise.

\section{Safety precautions}

An elbow exoskeleton supports physiological movements, taking into consideration the current health status of the patients, the goals of the therapy, etc. The most important factors which influence patient and therapist safety that we took into consideration during the development of the elbow exoskeleton were as follows:

- the method of wearing and repeatedly fixing the exoskeleton to the arm and elbow joint in order to avoid injuries;

- limitations of the personal range of motion (ROM);

- regulation and limitation of personal speed of movement;

- an emergency switch;

- protection against unauthorized use;

- safety alerts, low-battery and error signals; and

- online help desk and availability of service.

The potential for robots constraining the natural movement of the shoulder joint to cause subluxation at the shoulder was described by Jeong-Ho et al., but this does not apply in the case of an elbow exoskeleton. ${ }^{27}$ 
An important issue, especially in elderly patients, is the acceptance of advanced devices by the patients, their families and caregivers. It should be facilitated by reasonable and patient-adjusted learning of novel technologies.

\section{Material and methods}

\section{Design of elbow exoskeleton}

It has been decided that it will be sufficient to use a SDOF solution in constructing the prototype of an elbow exoskeleton for rehabilitation purposes. The simplicity of the actuation mechanism was set as one of the priorities in the design; thus, a single-axis stepper motor with a controller was found to be adequate for providing a reliable and precise source of motion for the exoskeleton, in contrast to the bionic model of the human arm, which requires an antagonistic actuator control, resulting in a much more complex control system and possibly also a heavier device.

This paper presents 2 solutions for an elbow exoskeleton for rehabilitation:

- asymmetrical with a 1-side drive system (Fig. 1,2) ${ }^{29}$;

- symmetrical with a 2 -side drive system (Fig. 3,4 ).

The asymmetrical design of the upper limb exoskeleton (ULE) was presented as a virtual prototype (Fig. 1) and a functional prototype of ULE (Fig. 2). In order to ensure

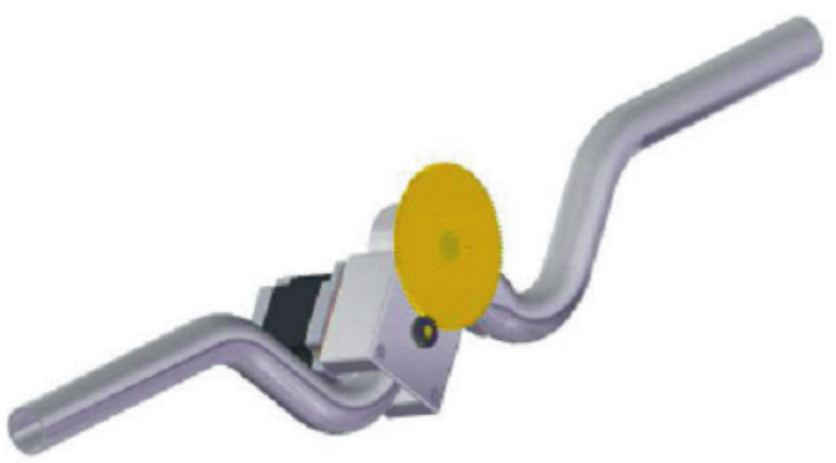

Fig. 1. Asymmetric upper limb exoskeleton (ULE) with a 1-side drive system: the virtual model made in Solid Edge (Siemens PLM Software, Plano, USA)

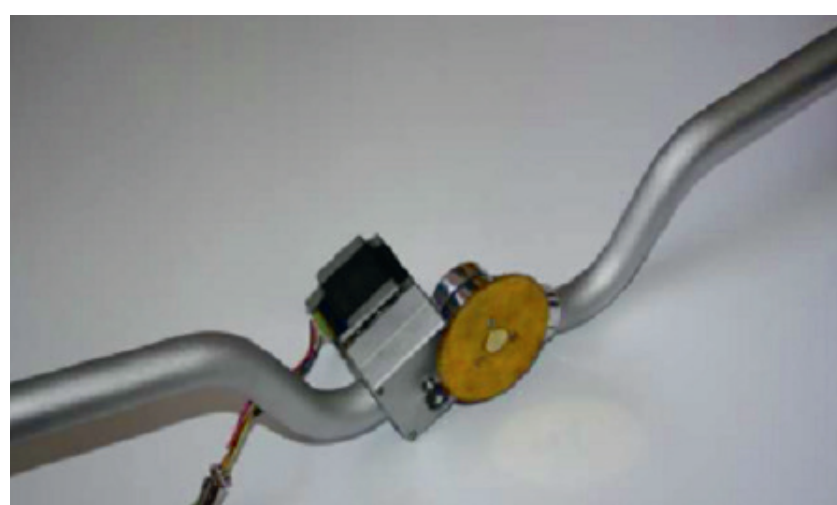

Fig. 2. Prototype of an asymmetrical upper limb exoskeleton (ULE) with a 1-side drive system

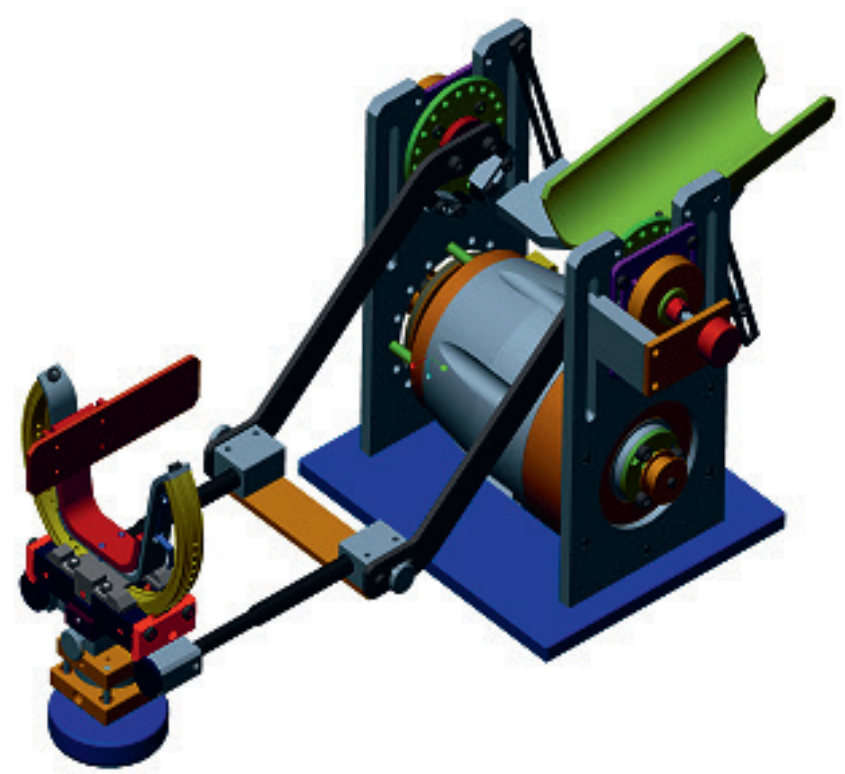

Fig. 3. Virtual model of a symmetrical upper limb exoskeleton (ULE) with 2-side drive system: upper arm (1) and wrist (2) supports

a strong and lightweight support structure, the exoskeleton frame was manufactured from aluminum.

Design work showed that locating the motor close to the elbow should provide the optimal functionality of the exoskeleton. A standard NEMA 23 stepper motor (MOONS', Shanghai, China) with a $1.8^{\circ}$ step size and a nominal torque of $1 \mathrm{Nm}$ was chosen for this project. To increase the torque, the motor was equipped with a helical gearbox with a 1:5 gear ratio, which effectively increased the available torque to $5 \mathrm{Nm}$. This provided for low weight and sufficient torque for joint actuation. The maximum peak torque output of $5 \mathrm{Nm}$ needs to be improved because it does not seem high enough to mobilize patients with no residual mobility. Spasticity can generate articulation torque higher than $5 \mathrm{Nm}$, preventing passive mobilization from the robot.

A virtual model of a symmetrical device is presented in Fig. 3. Its architecture consisted of 2 vertical side plates fixed to a base support, between which, in the lower part, a rotary motor is located. In the upper part of the plates, 2 parallel rods are hinged, which are able to link with the appendix to support the wrist.

As shown in Fig. 3, the patient puts his/her upper arm into the support positioned at the rear of the device and, using a special orthopedic glove, engages the wrist to the support positioned in front of the device. The wrist support has a semicircular guide and can rotate around its own axis, so as to allow the pronation-supination movement of the hand.

Integration (Fig. 4) of the symmetrical device was done by adopting $3 \mathrm{D}$-printed polymeric materials to create an ergonomic interface with the patients and by transferring the exoskeleton architecture around the arm of the patient to align the rotary joint of the device with the biomechanical rotary joint associated with the elbow of the patient. 

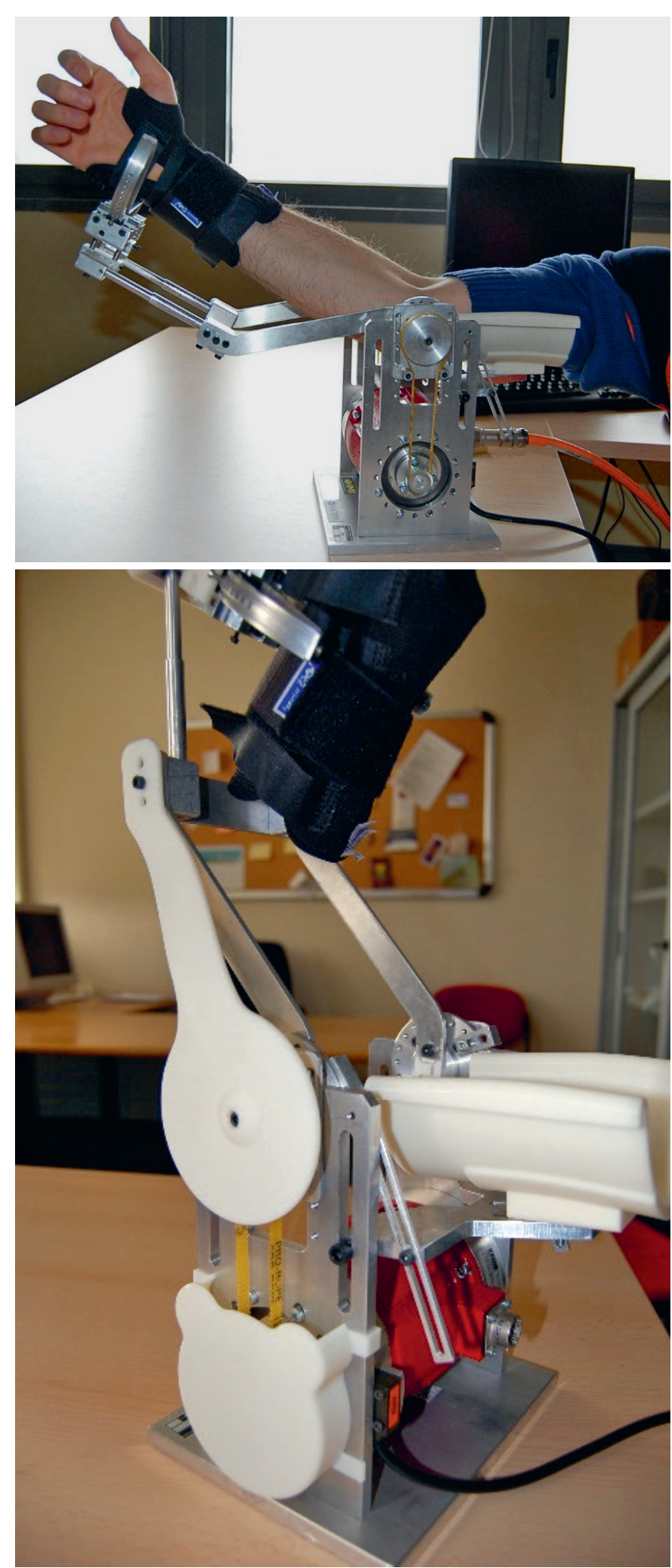

Fig. 4. Prototype of a symmetrical device with 2-side drive system: a) view of the device, b) view of 3D-printed ergonomic elements

\section{Exoskeleton control system}

The idea of the ULE control system is an asymmetrical design (Fig. 1,2). The exoskeleton control was created using a TB6560, a single-axis stepper motor driver control unit (Toshiba, Minata, Tokyo, Japan) (Fig. 5,6A) connected to the computer through an LPT port (Fig. 6B). A limit

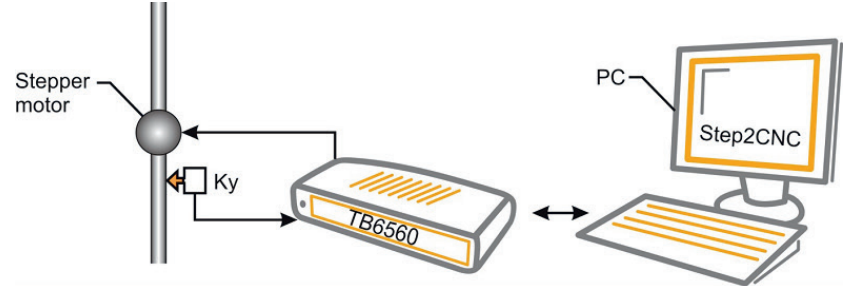

Fig. 5. Upper limb exoskeleton (ULE) control system with a single-axis stepper motor Ky - rotary encoder for stepper motor; Step2CNC - software for the controller; TB6560 - single-axis stepper motor driver control unit.

switch was used for reliable system start-up with a known position (Ky).

The system is powered with an industrial $600 \mathrm{~W}$ power supply (Power Control Systems, Veneto, Italy) providing a regulated output voltage up to $36 \mathrm{~V}$ and an output current of 16 A. A Dell GM 520 computer (Dell Computer Corporation, Round Rock, USA) (Fig. 6B) was used for control because of its available LPT port. In addition, this computer model has many USB ports, which makes it suitable for interfacing with other hardware. A touch LED screen (Fig. 6C) was used for easy interfacing with ULE.

The software interface for the controller was provided by Step2CNC v. 2.51 (Akcesoria CNC Elżbieta Taraszkiewicz, Augustów, Poland). ${ }^{30}$ It is mainly designed for numerical controlling of machining stations utilizing stepper motors. The main screen of the application is presented in Fig. 7. This software allows for both manual and automatic control using G-code. G-code can be imported from an external file or it can be edited directly in the Step2CNC software. ${ }^{31,32}$ The software was calibrated to use angular position as input. The position and the speed of movement can be controlled. G-code simplifies the creation of even complex motion patterns for training.

Using Step2CNC software enables complex control of the position and velocity of the motion path throughout the whole range. Motion can be prescribed as a function of $f$, which can later be converted to G-code. Positioning accuracy is dependent on the stepper motor step size. For a standard $1.8^{\circ}$ step size, 200 steps per revolution allow for more than sufficient control accuracy for a humanmachine interaction. The gearbox used in the design with a 1:5 gear ratio allowed the positioning accuracy to be increased to $0.36^{\circ}$ in full-step mode. The controller we used also allows operation in micro-stepping modes up to $1 / 16$ of a step. Step division can be selected using jumper switches available inside the casing. Figure 8 illustrates the positioning accuracy which can be obtained using different step size settings with and without an external gearbox.

\section{Results}

This study presents the original concept of an automatic control system based on Step2CNC software for the ULE (Fig. 7). The system consists of a limited number of commands 
a)

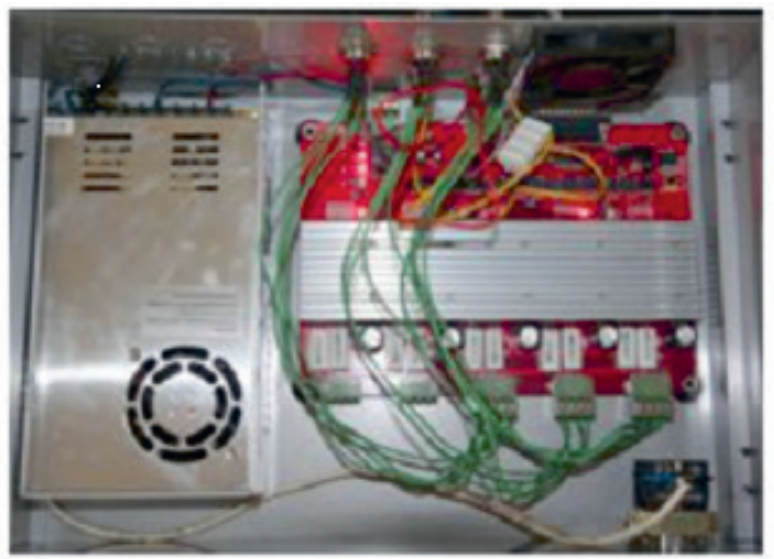

b)

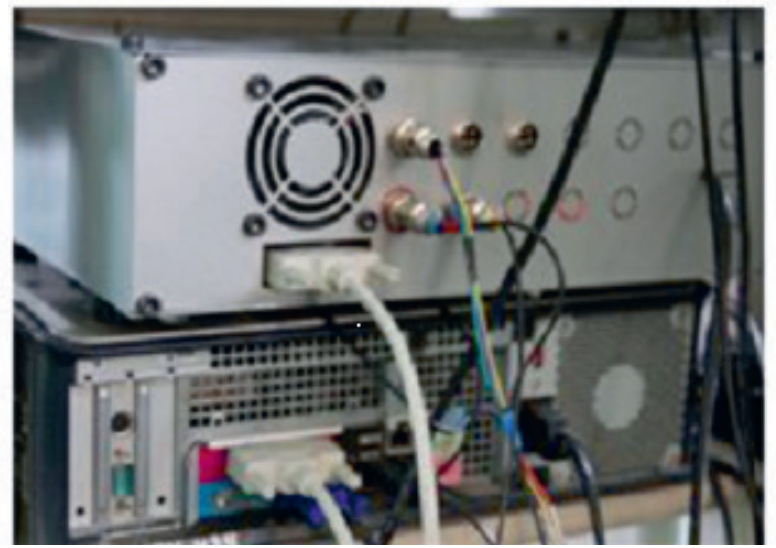

c)

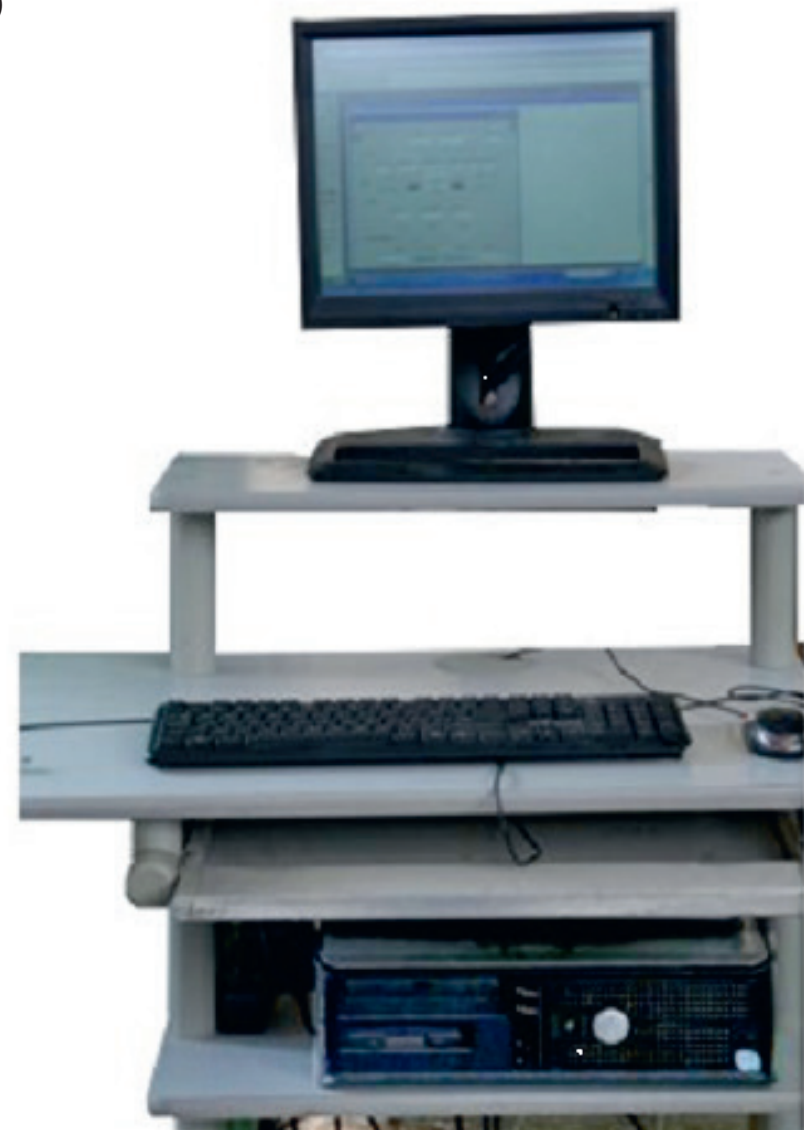

Fig. 6. Mobile control system

a - interior of the stepper motor controller; b - stepper motor controller and PC connection; c - complete station.

used to describe the movement trajectory and speed in an absolute coordinate system. The software includes visualization of the movement as a function of time. The limit switch position is also visualized. Despite the limitations of the visualization, the software is useful for the ULE control.

The following actions are possible for the ULE:

- clockwise motion of the motor (arm flexion);

- counterclockwise rotation of the motor (arm extension);

- stoppage of the motor for a specified time.

Figure 9 presents a definition of the control parameters of arm rotation.

Forearm motion speed can be individually predefined for each motion segment, which can be defined as fast movement speed or exercise speed. In case of exercise speed, it can be adjusted freely. Fast movement is performed at 1 predefined speed.

The following G-codes are used for the proposed system:

- G00 - fast motion with 1 predefined speed;

- G01 - exercise motion - the speed of motion can be freely defined on the control panel;

- G04 Hpar - a break in motion for a defined length of time, for instance, G04 H200 stops motion for 200 ms;

- M04 - works analogically to G04, using 0 as a delay parameter: the program is stopped until the "continue" key is pressed on the software interface;

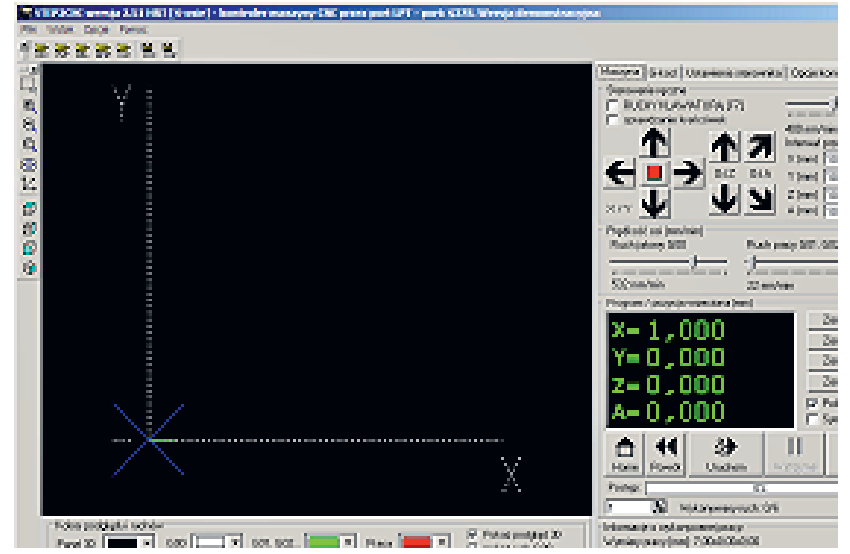

Fig. 7. Step2CNC user interface

- commands G00 and G01 can accept the following parameters:

- Xpar - $1^{\text {st }}$ axis coordinate;

- Ypar - $2^{\text {nd }}$ axis coordinate;

- Fpar - speed setting in $\mathrm{mm} / \mathrm{min}$.

- M30 - ends the program;

- G90 - enables absolute positioning;

- G91 - switches to relative positioning;

- G28 - returns to the home switch position. 


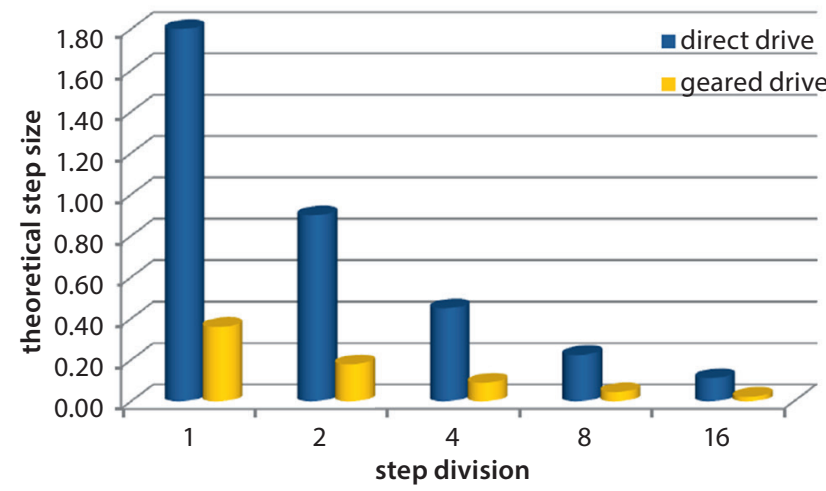

Fig. 8. Influence of step size on theoretical positioning accuracy with and without a gearbox

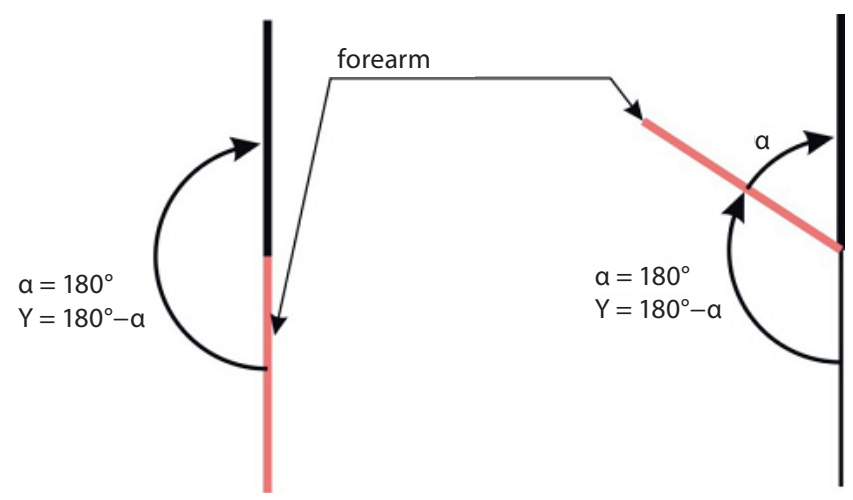

Fig. 9. Angle computation for the Step2CNC

$\mathrm{Y}$ - the angle parameter.

Even though only a single axis is controlled, the $\mathrm{X}$ parameter is used for visualization of the movement on the software interface. The G04 command can be used to pause the motion to let the patient rest between exercise sequences.

The velocity of the motion, $f y$, can be defined using the following relationship:

$$
f y=\frac{D y}{T} \times 60,
$$

where $D y$ is the rotation angle of the forearm and $T$ is the rotation time in seconds. Break time, $T_{b}$, which is a parameter of the G04 and M04 commands, needs to be expressed in miliseconds, thus:

$$
T_{b}=T \times 1000,
$$

where $T$ is the break time expressed in seconds.

Exemplary G-code is presented in Table 1. The sample code does not include the $\mathrm{X}$ parameter used only for visualization of the movement.

The presented code has been tested with the forearm exoskeleton. The formation of motion loops requires the same code fragment to be copied multiple times. Based on the example G-code and available commands, an automatic G-code generator was created according to the algorithm presented in Fig. 10.

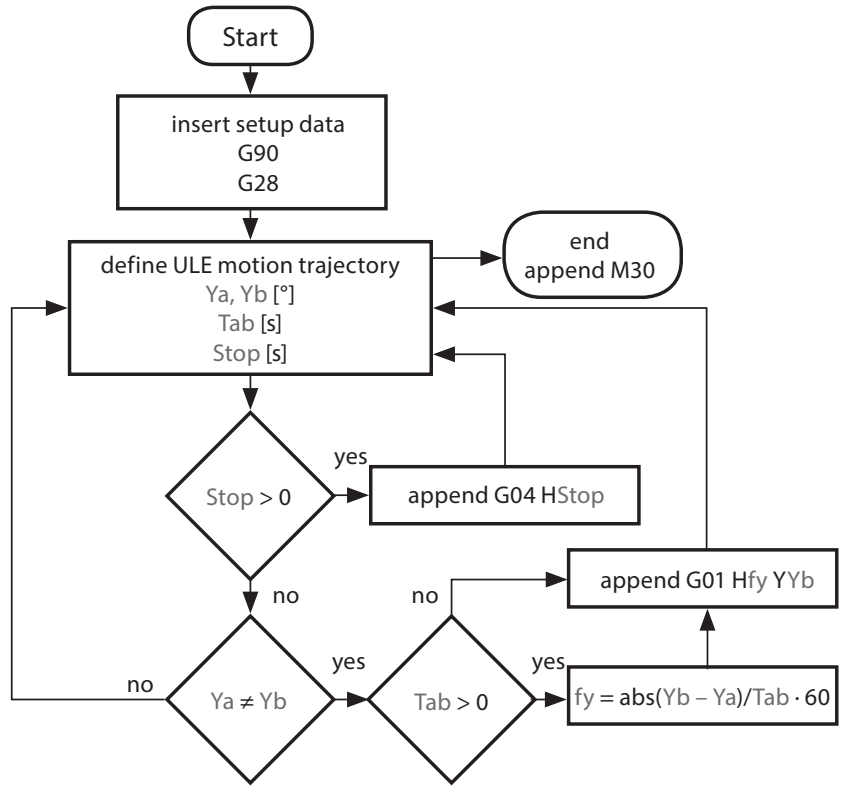

Fig. 10. Simplified algorithm of G-code generation

Ya, Yb - initial and final angles, Stop - pause time, Tab - motion time, fy - motion speed; ULE - upper limb exoskeleton.

The algorithm has been implemented in the custom Visual Basic 6 (Microsoft Corp., Redmond, USA) code CodeMake software (Fig. 11). The tool allows for simple G-code generation based on an input motion pattern. The software visualizes computation results and G-code output during operation. The text field in the software allows for easy copypasting of the code into Step2CNC. In addition, the generated code can be saved in a file. The CodeMake software contains a preview from a USB camera. It also has the "on top" feature, which locks the window in front of other windows, including Step2CNC. Such a configuration is convenient while using the remote control of Step2CNC, for instance, TeamViewer or Remote Desktop. ${ }^{33}$

\begin{tabular}{|c|c|}
\hline G-code & Description \\
\hline ULE control file & $\begin{array}{l}\text { file header - lines preceded with a semicolon are } \\
\text { treated as comments }\end{array}$ \\
\hline G90 & switches on absolute positioning mode \\
\hline G28 Y & $\begin{array}{l}\text { homing the arm unit limit switch is reached } \\
\text { to initialize the system with the arm position }\end{array}$ \\
\hline G01 F100 Y150 & $\begin{array}{l}\text { rotates the forearm } 100 \% \text { min until reaching } \\
\text { an absolute position of } 150^{\circ}\end{array}$ \\
\hline G04 H0 & $\begin{array}{l}\text { pauses the program execution until } \\
\text { the "continue" button is pressed }\end{array}$ \\
\hline G01 F20 Y60 & $\begin{array}{l}\text { slow motion with a velocity of } 20 \% \mathrm{~min} \text { until } \\
\text { an angular position of } 60^{\circ} \text { is reached }\end{array}$ \\
\hline G04 H2000 & $\begin{array}{l}\text { pauses program for } 2 \mathrm{~s} \text { and then continues } \\
\text { automatically }\end{array}$ \\
\hline G01 F50 Y0 & $\begin{array}{l}\text { sets motion at a velocity of } 50 \% \text { min until initial } \\
\text { position }\left(0^{\circ}\right) \text { is reached }\end{array}$ \\
\hline M30 & ends program \\
\hline
\end{tabular}

Table 1. Example of G-code

ULE - upper limb exoskeleton. 

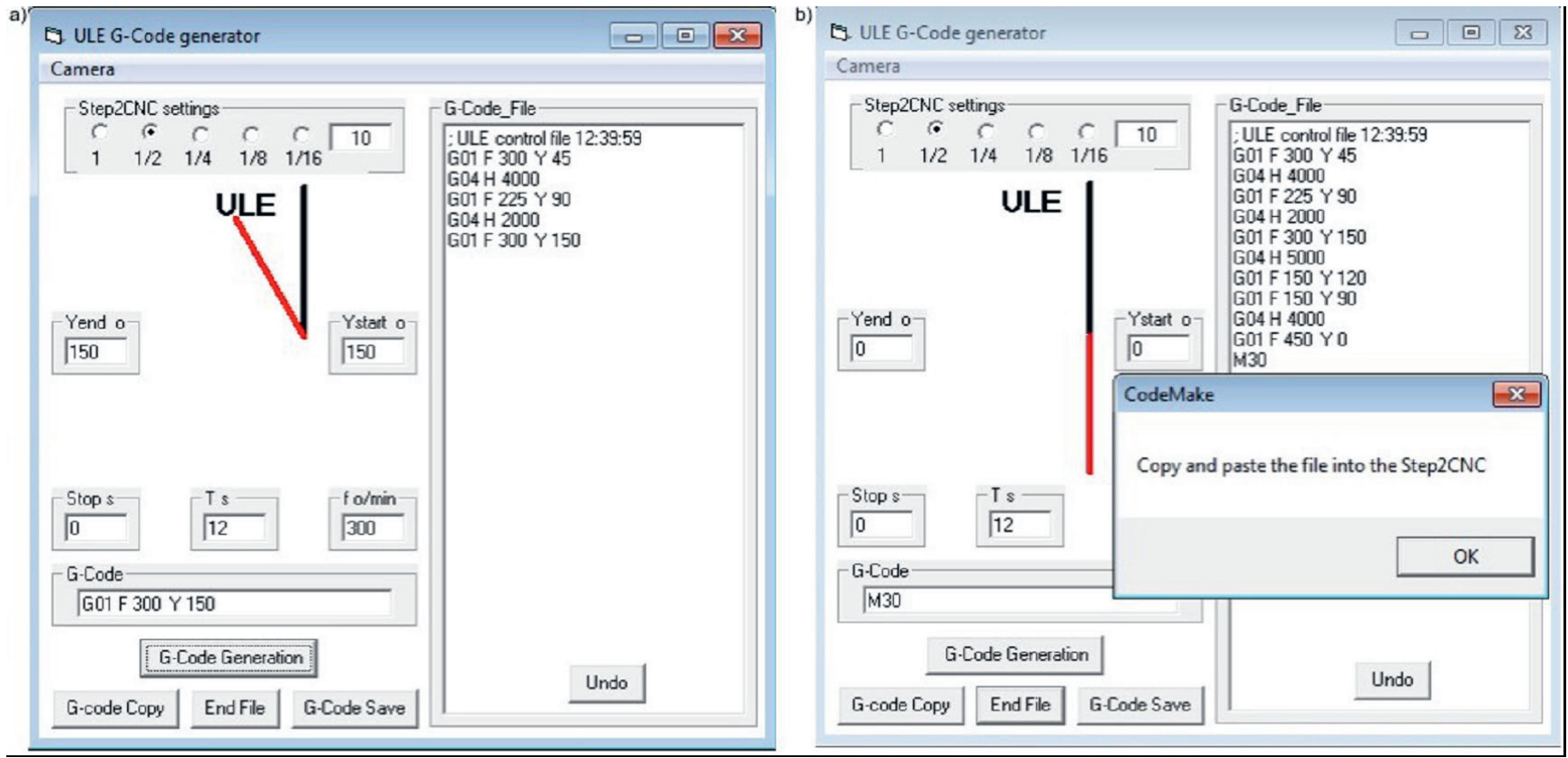

Fig. 11. Main window of the CodeMake tool: a) during control code generation; b) when the G-code is ready

During remote operation (Fig. 12,13), there is an option of adjusting the camera resolution to provide smooth image transmission even in the case of slow Internet connection. Remote operation mode allows the user to create exercise programs and to supervise exercise performance even if the patient and the operator are at distant locations.

Quantitative analysis showed significant benefits from an economic point of view (lower price and wider accessibility) and from a logistical one (easier manufacturing, $3 \mathrm{D}$-printing potential and reverse engineering for customized solutions).

\section{Discussion}

Despite several decades of work on exoskeletons, significant scientific contributions in the applications of rehabilitation and functional compensation and substitution have only begun to appear in the last 10 years. As exoskeletons are characterized by close cognitive and physical interaction with the human user, the requirements applied to the cognitive interaction are strict. As technological advances are made, there is much potential for growth in this field.

The evidence supporting the upper limb rehabilitation using robotics to facilitate therapeutic process makes robotic control systems a significant emerging field in robotics, biocybernetics, rehabilitation engineering, and clinical medicine. It becomes more complex and integral, taking into consideration the International Classification of Functioning (ICF) perspective to correctly evaluate the disabling effect of neuromotor disorders. Prior to the year 2000, there was a paucity of high-quality evidence regarding the management of neuromotor disorders with elbow

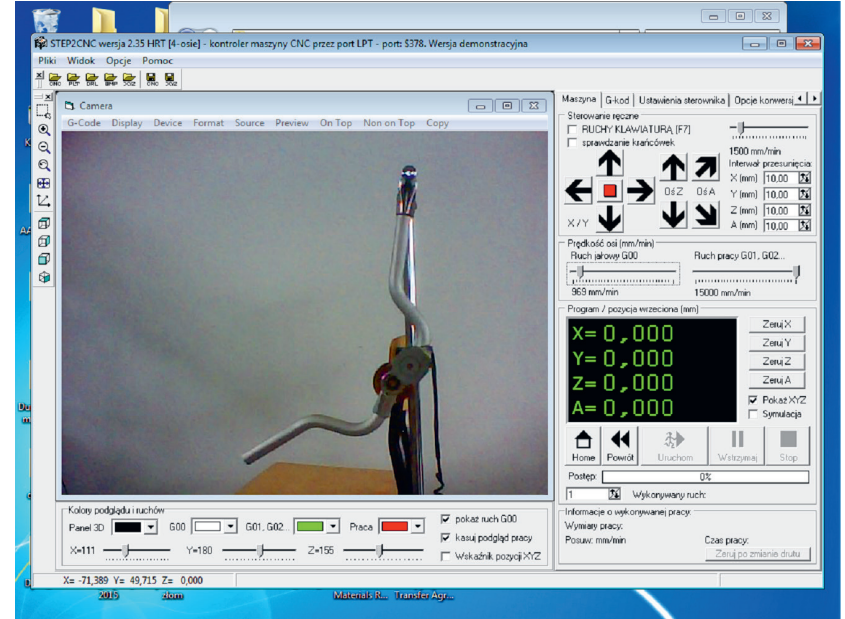

Fig. 12. Remote view of the Step2CNC and CodeMake window on top with a live preview of upper limb exoskeleton (ULE)

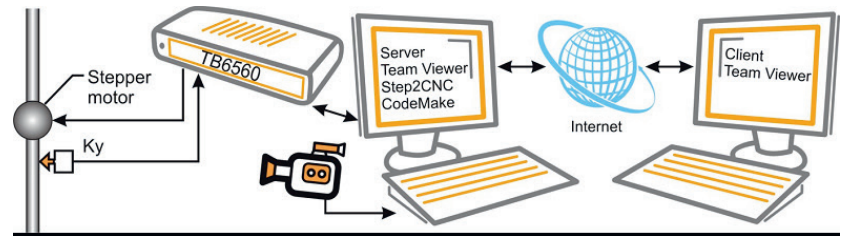

Fig. 13. System setup for upper limb exoskeleton (ULE) remote control

Ky - Ky encoder for motor control purposes.

exoskeletons. At one time, practitioners might have believed that elbow neurorehabilitation with such an exoskeleton was not effective because of the lack of empirical evidence. Robot-aided rehabilitation of the upper limbs is still a complementary therapy method, even as a home-based 
rehabilitation treatment. There is still a lack of evidence that robotic therapy is more effective than traditional face-to-face treatment. Strong evidence can be obtained using randomized controlled trials, large patient samples and a control group for comparison. However, shortages in the area of specialized personnel (including physicians, physiotherapists, nurses, etc.) make the robotic solution cheaper and more accessible. Easily transportable, wearable devices could improve rehabilitation after discharge as well, in outpatient or home-based settings. Efforts are being made to establish the ideal type of treatment, length of training and patient's characteristics for a successful treatment of this type. Cost-effective solutions, reduced effective hospitalization, early discharge, and home-based, long-term rehabilitation make robotic systems a new, basic and cheaper modality. ${ }^{34,35}$

The advantages of the proposed exoskeleton reported here reflect current state-of-the-art. A proposed control strategy relies on a closed-loop position control, performance, low manufacturing costs, and predictable performance in a rehabilitation setting. All these factors play an important role in establishing the directions for further research, e.g., integrated force sensors in the device, measurements of torque interactions on the elbow joint, and assessment and response to an overload of articulation.

The aforementioned issues describe the main limitations of the current study.

Based on a literature review and our own experience during working on the proposed system, we can formulate the following conclusions.

The designed actuation system utilizing a stepper motor and a special gearbox provides precise positioning and repeatability of movement (angular accuracy within $0.18-0.36^{\circ}$ ), a wide range of speeds, and full torque availability even at 0 speed (as long as there is power in the windings).

Step2CNC software offers a simple software-hardware interface, providing G-code interpretation and control strategy; it also allows the user to edit G-code, thus providing an option for code sequence generation. The aluminum construction of the exoskeleton yields a strong and lightweight system $(2 \mathrm{~kg})$.

The prototype consisted of easily obtainable components, with a simple and off-the-shelf control system.

The prepared Code-Make software allows for camera image previews, useful for remote operation, as well as for straightforward G-code generation concerning desired positions and delays in the exercise program.

Remote operation of the system is possible and easy to implement using Remote Desktop or Team Viewer.

The control system presented above uses a standard G-code implementation as well as a typical Computer $\mathrm{Nu}$ merical Control (CNC). Therefore, it is possible to expand this system by adding additional degrees of freedom.

The limitations of the study are the imperfections observed in the construction of the exoskeleton and its shortcomings in terms of mapping all the movements of the elbow and forearm. In this respect, the exoskeleton must be improved. Another limitation is the fact that this is only a theoretical paper with no clinical studies. Further research should include deeper clinical studies on large samples of patients. Such an approach allows for further compartmental studies and better fulfillment of the needs of the patients, their families and caregivers, as well as therapists.

\section{Conclusions}

The current study suggests that choosing an elbow exoskeleton may have not only clinical but also possible economic and logistical advantages. In the future, exoskeleton-based rehabilitation of upper limb function may constitute the most promising therapeutic tool which can meet the increasing demand for therapy.

The design of ULE presented here is a good solution for the rehabilitation of the patient. The components of the control system are easy obtainable and can be used for control of a ULE by the available application, Step2CNC. CodeMake software allows for a camera image preview, is useful for remote operation and can generate G-code straightforwardly.

\section{References}

1. Kiguchi K, Kariya S, Watanabe K, Izumi K, Fukuda T. An exoskeletal robot for human elbow motion support-sensor fusion, adaptation, and control. IEEE Trans Syst Man Cybern B Cybern. 2001;31(3):353-361.

2. Lo AC, Guarino PD, Richards LG, et al. Robot-assisted therapy for long-term upper-limb impairment after stroke. N Engl J Med. 2010;362(19):1772-1783.

3. Lum P, Reinkensmeyer D, Mahoney R, Rymer WZ, Burgar C. Robotic devices for movement therapy after stroke: Current status and challenges to clinical acceptance. Top Stroke Rehabil. 2002;8(4):40-53.

4. Marchal-Crespo L, Reinkensmeyer DJ. Review of control strategies for robotic movement training after neurologic injury. J Neuroeng Rehabil. 2009;6:20.

5. Takahashi CD, Der-Yeghiaian L, Le V, Motiwala RR, Cramer SC. Robotbased hand motor therapy after stroke. Brain. 2008;131(Pt 2):425-437.

6. Howard A, Brooks D, Brown E, Gebregiorgis A, Chen YP. Non-contact versus contact-based sensing methodologies for in-home upper arm robotic rehabilitation. IEEE Int Conf Rehabil Robot. 2013;2013: 6650487.

7. Brochard S, Robertson J, Médée B, Rémy-Néris O. What's new in new technologies for upper extremity rehabilitation? Curr Opin Neurol. 2010;23(6):683-687.

8. Li C, Rusák Z, Horváth I, Ji L. Influence of complementing a robotic upper limb rehabilitation system with video games on the engagement of the participants: A study focusing on muscle activities. Int J Rehabil Res. 2014;37(4):334-342.

9. Tsagarakis NG, Caldwell DG. Development and control of a "softactuated" exoskeleton for use in physiotherapy and training. Auton Robots. 2003;15(1):21-33.

10. Pons JL. Rehabilitation exoskeletal robotics. The promise of an emerging field. IEEE Eng Med Biol Mag. 2010;29(3):57-63.

11. Taveggia G, Villafañe JH, Vavassori F, Lecchi C, Borboni A, Negrini S. Multimodal treatment of distal sensorimotor polyneuropathy in diabetic patients: A randomized clinical trial. J Manipulative Physiol Ther. 2014;37(4):242-252.

12. Fausti D, Petrogalli G, Villafañe JH, Faglia R. Study, design and preliminary tests of an automatic device for elbow rehabilitation. Appl Mech Mater. 2015;783:1-15.

13. Nycz CJ, Delph MA, Fischer GS. Modeling and design of a tendon actuated soft robotic exoskeleton for hemiparetic upper limb rehabilitation. Conf Proc IEEE Eng Med Biol Soc. 2015:3889-3892. 
14. Taveggia G, Borboni A, Mule C, Villafañe JH, Negrini S. Conflicting results of robot-assisted versus usual gait training during postacute rehabilitation of stroke patients: A randomized clinical trial. Int J Rehabil Res. 2016;39(1):29-35.

15. Bishop L, Stein J. Three upper limb robotic devices for stroke rehabilitation: A review and clinical perspective. NeuroRehabilitation. 2013;33(1):3-11.

16. Hochstenbach-Waelen A, Seelen HA. Embracing change: Practical and theoretical considerations for successful implementation of technology assisting upper limb training in stroke. J Neuroeng Rehabil. 2012;9:52.

17. Loureiro RC, Harwin WS, Nagai K, Johnson M. Advances in upper limb stroke rehabilitation: A technology push. Med Biol Eng Comput. 2011;49(10):1103-1118.

18. Lu EC, Wang RH, Hebert D, Boger J, Galea MP, Mihailidis A. The development of an upper limb stroke rehabilitation robot: Identification of clinical practices and design requirements through a survey of therapists. Disabil Rehabil Assist Technol. 2011;6(5):420-431.

19. Maciejasz P, Eschweiler J, Gerlach-Hahn K, Jansen-Troy A, Leonhardt S. A survey on robotic devices for upper limb rehabilitation. J Neuroeng Rehabil. 2014;11:3.

20. Morales R, Badesa FJ, Garcia-Aracil N, Sabater JM, Perez-Vidal C. Pneumatic robotic systems for upper limb rehabilitation. Med Biol Eng Comput. 2011;49(10):1145-1156.

21. Huang LL, Lee CF, Hsieh CL, Chen MH. Upper extremity rehabilitation equipment for stroke patients in Taiwan: Usage problems and improvement needs. Occup Ther Int. 2013;20(4):205-214.

22. Sukal-Moulton T, Krosschell KJ, Gaebler-Spira DJ. Dewald JP. Motor impairment factors related to brain injury timing in early hemiparesis. Part I: Expression of upper-extremity weakness. Neurorehabil Neural Repair. 2014;28(1):13-23.

23. Zackowski KM, Dromerick AW, Sahrmann SA, Thach WT, Bastian AJ. How do strength, sensation, spasticity and joint individuation relate to the reaching deficits of people with chronic hemiparesis? Brain. 2004;127(Pt 5):1035-1046.
24. Mottram CJ, Suresh NL, Heckman CJ, Gorassini MA, Rymer WZ. Origins of abnormal excitability in biceps brachii motoneurons of spastic-paretic stroke survivors. J Neurophysiol. 2009;102(4):2026-2038.

25. Gracies JM. Pathophysiology of spastic paresis. Il: Emergence of muscle overactivity. Muscle Nerve. 2005;31(5):552-571.

26. Brunnstrom S. Movement Therapy in Hemiplegia: A Neurophysiological Approach. New York, NY: Harper \& Row; 1970.

27. Jeong-Ho P, Kyoung-Soub L, Kyeong-Hun J, Dong-Hyun K, HyungSoon P. Low cost and light-weight multi-DOF exoskeleton for comprehensive upper limb rehabilitation. Proceedings of 11th Conference on Ubiquitous Robots and Ambient Intelligence (URAI). 2014:138-139.

28. Vitiello N, Lenzi T, Roccello S, et al. NEUROExos: A powered elbow exoskeleton for physical rehabilitation. IEEE Trans. Robot. 2013;29(1): 220-235.

29. Mikołajczyk T, Olaru A, Walkowiak P. Upper limb exoskeleton controlled by stepper motor. Applied Mechanics and Materials. 2015;811:305-310.

30. Step2CNC. https://www.ebmia.pl/step2cnc-p-11637.html. Updated January 5, 2015. Accessed January 5, 2015.

31. Mikołajczyk T, Borboni A, Mackowski D, Matuszewski M. Example of tool with two numerical controlled axes. Applied Mechanics and Materials. 2015;772:224-229.

32. Mikołajczyk T, Dorsz D, Romanowski L. Design and control system of parallel kinematics manipulator. Applied Mechanics and Materials. 2013;436:390-339.

33. TeamViewer. https://www.teamviewer.com/pl/download/windows/. Updated January 5, 2015. Accessed January 5, 2015.

34. Poli P, Morone G, Rosati G, Masiero S. Robotic technologies and rehabilitation: New tools for stroke patients' therapy. Biomed Res Int. 2013:153872.

35. Masiero S, Poli P, Rosati G, et al. The value of robotic systems in stroke rehabilitation. Expert Rev Med Devices. 2014;11(2):187-198. 